\title{
The many-body exchange-correlation hole at metal surfaces
}

\author{
Lucian A. Constantin ${ }^{1}$ and J. M. Pitarke ${ }^{2,3}$ \\ ${ }^{1}$ Department of Physics and Quantum Theory Group, \\ Tulane University, New Orleans, LA 70118 \\ ${ }^{2}$ CIC nanoGUNE Consolider, Tolosa Hiribidea 76, \\ E-20018 Donostia - San Sebastian, Basque Country \\ ${ }^{3}$ Materia Kondentsatuaren Fisika Saila (UPV/EHU), \\ DIPC, and Centro Física Materiales (CSIC-UPV/EHU), \\ 644 Posta kutxatila, E-48080 Bilbo, Basque Country
}

(Dated: July 12, 2021)

\begin{abstract}
We present a detailed study of the coupling-constant-averaged exchange-correlation hole density at a jellium surface, which we obtain in the random-phase approximation (RPA) of many-body theory. We report contour plots of the exchange-only and exchange-correlation hole densities, the integration of the exchange-correlation hole density over the surface plane, the on-top correlation hole, and the energy density. We find that the on-top correlation hole is accurately described by local and semilocal density-functional approximations. We also find that for electrons that are localized far outside the surface the main part of the corresponding exchange-correlation hole is localized at the image plane.
\end{abstract}

PACS numbers: 71.10.Ca,71.15.Mb,71.45.Gm

\section{INTRODUCTION}

The exchange-correlation (xc) energy of a manyelectron system is the only density functional that has to be approximated in the Kohn-Sham (KS) formalism of

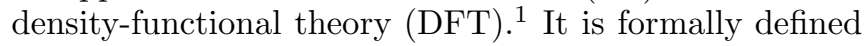
by the following equation derived from the HellmannFeynman theorem: $\underline{\underline{2}}$

$$
E_{x c}[n]=\frac{1}{2} \int d \mathbf{r} \int d \mathbf{r}^{\prime} \int_{0}^{1} d \lambda \frac{\rho_{2}^{\lambda}\left(\mathbf{r}^{\prime}, \mathbf{r}\right)}{\left|\mathbf{r}-\mathbf{r}^{\prime}\right|}-U[n],
$$

where $n(\mathbf{r})$ is the density of a spin-unpolarized system of $N$ electrons, $U[n]=(1 / 2) \int d \mathbf{r} n(\mathbf{r}) n\left(\mathbf{r}^{\prime}\right) /\left|\mathbf{r}-\mathbf{r}^{\prime}\right|$ is the Hartree energy, and $\rho_{2}^{\lambda}\left(\mathbf{r}^{\prime}, \mathbf{r}\right)$ is the reduced two-particle density matrix

$$
\begin{aligned}
\rho_{2}^{\lambda}\left(\mathbf{r}^{\prime}, \mathbf{r}\right)= & N(N-1) \sum_{\sigma, \sigma^{\prime}, \ldots, \sigma_{N}} \int d \mathbf{r}_{3} \ldots d \mathbf{r}_{N} \\
& \times\left|\Psi^{\lambda}\left(\mathbf{r}^{\prime} \sigma^{\prime}, \mathbf{r} \sigma, \mathbf{r}_{3} \sigma_{3}, \ldots, \mathbf{r}_{N} \sigma_{N}\right)\right|^{2} .
\end{aligned}
$$

Here, $\Psi^{\lambda}\left(\mathbf{r}_{1} \sigma_{1}, \ldots, \mathbf{r}_{N} \sigma_{N}\right)$ is the antisymmetric wavefunction that yields the density $n(\mathbf{r})$ and minimizes the expectation value of $\hat{T}+\lambda \hat{V}_{e e}$, where $\hat{T}=-\sum_{i=1}^{N} \nabla_{i}^{2} / 2$ and $\hat{V}_{e e}=\frac{1}{2} \sum_{i} \sum_{j \neq i} \frac{1}{\left|\mathbf{r}_{i}-\mathbf{r}_{j}\right|}$ are the kinetic energy and the electron-electron interaction operators. Eq. (2) shows that $\rho_{2}^{\lambda}\left(\mathbf{r}^{\prime}, \mathbf{r}\right) d \mathbf{r}^{\prime} d \mathbf{r}$ is the joint probability of finding an electron of arbitrary spin in $d \mathbf{r}^{\prime}$ at $\mathbf{r}^{\prime}$ and an electron of arbitrary spin in $d \mathbf{r}$ at $\mathbf{r}$, assuming that the Coulomb interaction is $\lambda /\left|\mathbf{r}-\mathbf{r}^{\prime}\right|$. In the case of noninteracting KS electrons (i.e., $\lambda=0), \rho_{2}^{\lambda=0}\left(\mathbf{r}^{\prime}, \mathbf{r}\right)$ is the exchange-only reduced two-particle density matrix that is expressible in terms of KS orbitals. (Unless otherwise stated, atomic units are used throughout, i.e., $e^{2}=\hbar=m_{e}=1$.)

Hence, the xc energy can be expressed as the electrostatic interaction between individual electrons and the corresponding (and sorrounding) coupling-constantaveraged xc hole density $\bar{n}_{x c}\left([n] ; \mathbf{r}, \mathbf{r}^{\prime}\right)$, as follows

$$
E_{x c}[n]=\int d \mathbf{r} e_{x c}(\mathbf{r})=\frac{1}{2} \int d \mathbf{r} \int d \mathbf{r}^{\prime} \frac{n(\mathbf{r}) \bar{n}_{x c}\left([n] ; \mathbf{r}, \mathbf{r}^{\prime}\right)}{\left|\mathbf{r}-\mathbf{r}^{\prime}\right|}
$$

where [see Eqs. (11) and (3)]:

$$
\bar{n}_{x c}\left([n] ; \mathbf{r}, \mathbf{r}^{\prime}\right)=\frac{1}{n(\mathbf{r})} \int_{0}^{1} d \lambda \rho_{2}^{\lambda}\left(\mathbf{r}^{\prime}, \mathbf{r}\right)-n\left(\mathbf{r}^{\prime}\right),
$$

and $e_{x c}(\mathbf{r})$ is the xc energy density. The xc hole density $n_{x c}\left([n] ; \mathbf{r}, \mathbf{r}^{\prime}\right)$ is the result of three effects: self-interaction correction to the Hartree approximation, Pauli exclusion principle, and the electron correlation due to Coulomb repulsion between electrons.

The adiabatic-connection fluctuation-dissipation theorem provides an elegant path to the exact couplingconstant-averaged xc hole density,,$\frac{3,4,5,6}{6}$ which can be written as follows ${ }^{7}$

$$
\begin{array}{r}
\bar{n}_{x c}\left([n] ; \mathbf{r}, \mathbf{r}^{\prime}\right)=\frac{1}{n(\mathbf{r})}\left[-\frac{1}{\pi} \int_{0}^{\infty} d \omega \int_{0}^{1} d \lambda \chi^{\lambda}\left(\mathbf{r}, \mathbf{r}^{\prime} ; \omega\right)\right. \\
\left.-n(\mathbf{r}) \delta\left(\mathbf{r}-\mathbf{r}^{\prime}\right)\right],
\end{array}
$$

where $\chi^{\lambda}\left(\mathbf{r}, \mathbf{r}^{\prime} ; \omega\right)$ is the density-response function of the interacting system at coupling strength $\lambda$ and satisfies, in the framework of time-dependent density-functional theory (TDDFT), the following exact Dyson-type equation ${ }^{8}$

$$
\begin{aligned}
& \chi_{\lambda}\left(\mathbf{r}, \mathbf{r}^{\prime} ; \omega\right)=\chi_{0}\left(\mathbf{r}, \mathbf{r}^{\prime} ; \omega\right)+\int d \mathbf{r}_{1} d \mathbf{r}_{2} \chi_{0}\left(\mathbf{r}, \mathbf{r}_{1} ; \omega\right) \\
\times & \left\{\frac{\lambda}{\left|\mathbf{r}_{1}-\mathbf{r}_{2}\right|}+f_{x c, \lambda}[n]\left(\mathbf{r}_{1}, \mathbf{r}_{2} ; \omega\right)\right\} \chi_{\lambda}\left(\mathbf{r}_{2}, \mathbf{r}^{\prime} ; \omega\right) .
\end{aligned}
$$

Here, $\chi_{0}\left(\mathbf{r}, \mathbf{r}^{\prime} ; \omega\right)$ is the density-response function of non-interacting KS electrons (which is exactly 
known in terms of $\mathrm{KS}$ orbitals $\left.{ }^{9}\right)$ and $f_{x c, \lambda}[n]\left(\mathbf{r}, \mathbf{r}^{\prime} ; \omega\right)$ is the Fourier transform with respect to time $\left[f_{x c, \lambda}[n]\left(\mathbf{r}, \mathbf{r}^{\prime} ; \omega\right)=\int_{-\infty}^{\infty} d t e^{i \omega t} f_{x c, \lambda}[n]\left(\mathbf{r}, t, \mathbf{r}^{\prime}, 0\right)\right]$ of the unknown $\lambda$-dependent xc kernel, formally defined by

$$
f_{x c, \lambda}[n]\left(\mathbf{r}, t, \mathbf{r}^{\prime}, t^{\prime}\right)=\frac{\delta v_{x c}^{\lambda}[n](\mathbf{r}, t)}{\delta n\left(\mathbf{r}^{\prime}, t^{\prime}\right)}
$$

where $v_{x c}^{\lambda}[n](\mathbf{r}, t)$ is the exact time-dependent xc potential of TDDFT. When $f_{x c, \lambda}[n]\left(\mathbf{r}, \mathbf{r}^{\prime} ; \omega\right)$ is taken to be zero, Eq. (6) reduces to the random phase approximation (RPA). If the interacting density response function $\chi_{\lambda}\left(\mathbf{r}, \mathbf{r}^{\prime} ; \omega\right)$ is replaced by the noninteracting KS densityresponse function $\chi_{0}\left(\mathbf{r}, \mathbf{r}^{\prime} ; \omega\right)$, then Eq. (5) yields the exchange-only hole density.

The scaling relation of the correlation hole density at coupling constant $\lambda^{10,11}$ leads to the following equation for the coupling-constant-averaged correlation hole density:

$$
\bar{n}_{c}\left([n] ; \mathbf{r}, \mathbf{r}^{\prime}\right)=\int_{0}^{1} d \lambda\left(\frac{\lambda}{w}\right)^{3} n_{c}^{w}\left(\left[n_{w / \lambda}\right], \frac{\lambda}{w} \mathbf{r}, \frac{\lambda}{w} \mathbf{r}^{\prime}\right),
$$

where $0<w<<1$ is a fixed constant, and $n_{\gamma}(\mathbf{r})=$ $\gamma^{3} n(\gamma \mathbf{r})$ is a uniformly-scaled density $\frac{12}{2}$ Eq. (8) shows that the whole many-body problem is equivalent to the knowledge of the universal correlation hole density at a small, fixed coupling strength $w$.

There is a "Jacob's ladder" 13 classification (in RPA and beyond RPA) of nonempirical approximations to the angle-averaged xc hole density

$$
\bar{n}_{x c}([n] ; \mathbf{r}, u)=\frac{1}{4 \pi} \int d \Omega \bar{n}_{x c}\left([n] ; \mathbf{r}, \mathbf{r}^{\prime}\right),
$$

where $d \Omega$ is the differential solid angle around the direction of $\mathbf{u}=\mathbf{r}^{\prime}-\mathbf{r}$. The simplest rung of the ladder is the local spin density approximation (LSDA) of the xc hole density $\bar{n}_{x c}\left(n_{\uparrow}, n_{\downarrow} ; u\right)$ that has as ingredients only the spin densities. (For the RPA-based LSDA $\mathrm{xc}$ hole and for the LSDA xc hole, see Refs. 14, 15 and Refs. 14, 16, 17, respectively.) The next rung is the generalized gradient approximation (GGA) xc hole density $\bar{n}_{x c}\left(n_{\uparrow}, n_{\downarrow}, \nabla n_{\uparrow}, \nabla n_{\downarrow}, u\right)$. (See Ref. 14 for the smoothed GGA exchange hole model, Ref. 18 for the PBE-GGA 19 correlation hole, and Ref. 15 for the RPAbased GGA hole model. For a GGA xc hole constructed for solids, see Ref. 20.) The third rung on this ladder is the non-empirical meta-GGA xc hole density 21 $\bar{n}_{x c}\left(n_{\uparrow}, n_{\downarrow}, \nabla n_{\uparrow}, \nabla n_{\downarrow}, \tau_{\uparrow}, \tau_{\downarrow}, u\right)$ that depends on spin densities and their gradients, as well as the positive KS kinetic energy densities $\tau_{\uparrow}$ and $\tau_{\downarrow}$, and that was constructed to satisfy many exact constraints. (For an RPA-based meta-GGA xc hole model, see also Ref. 21.)

Jellium is a simple model of a simple metal, in which the ion cores are replaced by a uniform positive background of density $\bar{n}=3 / 4 \pi r_{s}^{3}=k_{F}^{3} / 3 \pi^{2}$ and the valence electrons in the spin-unpolarized bulk neutralize this background. $r_{s}$ is the bulk density parameter and
$k_{F}$ is the magnitude of the bulk Fermi wavevector. At a jellium surface, the plane $z=0$ separates the uniform positive background $(z>0)$ from the vacuum $(z<0)$, and the electrons can leak out into the vacuum. This electron system is translationally invariant in the plane of the surface.

The exchange hole at a jellium surface was studied in Ref. 22 (using a finite linear-potential model ${ }^{23}$ ), and in Refs. 24 25 (using the infinite barrier model (IBM) ${ }^{26}$ ). The behavior of the xc hole at a jellium surface was investigated at the RPA level using IBM orbitals $\underline{27}$ Hence, existing calculations of the exchange-only and xc hole at a jellium surface invoke either a finite linear-potential model or the IBM for the description of single-particle orbitals. An exception is a self-consistent calculation of the RPA xc hole density reported briefly in Refs. 28 and 29, in which accurate LSDA single-particle orbitals were employed.

In this paper, we present extensive self-consistent calculations of the exact-exchange hole and the RPA xc hole at a jellium surface. We report contour plots of the corresponding hole densities, the integration of the xc hole density over the surface plane, and the on-top correlation hole. We find that the on-top RPA correlation hole $\bar{n}_{c}([n] ; \mathbf{r}, \mathbf{r})$ is accurately described by the on-top RPAbased LSDA hole, in accord with the work of Perdew et $a l^{5,30,31}$

\section{THE EXACT-EXCHANGE HOLE AND THE RPA XC HOLE AT A JELLIUM SURFACE}

Let us consider a jellium surface with the surface plane at $z=0$. Using its translational invariance in a plane perpendicular to the $z$ axis, the coupling-constant-averaged xc hole density of Eq. (5) can be written as follows $\underline{29}$

$$
\begin{aligned}
\bar{n}_{x c}\left([n] ; r, z, z^{\prime}\right) & =-\frac{1}{2 \pi} \int_{0}^{\infty} d q_{||} q_{||} J_{0}\left(q_{||} r\right)\left[\frac{1}{\pi n(z)} \int_{0}^{1} d \lambda \int_{0}^{\infty} d \omega\right. \\
& \left.\times \chi^{\lambda}\left(q_{\| \mid}, z, z^{\prime}, \omega\right)-\delta\left(z-z^{\prime}\right)\right],
\end{aligned}
$$

where $r=\left|\mathbf{r}_{\|}-\mathbf{r}_{\|}^{\prime}\right|$, and $\mathbf{q}_{\|}$is a two-dimensional (2D) wavevector. $\chi^{\lambda}\left(q_{\|}, z, z^{\prime}, \omega\right)$ represents the $2 \mathrm{D}$ Fourier transform of the interacting density response function of Eq. (6), which in the RPA is obtained by neglecting the xc kernel $f_{x c}$. The exact-exchange hole density is obtained by simply replacing in Eq. (10) $\chi^{\lambda}\left(q_{\|}, z, z^{\prime}, \omega\right)$ by the corresponding KS noninteracting density response function $\chi^{0}\left(q_{\|}, z, z^{\prime}, \omega\right)$.

For the evaluation of Eq. (10), we follow the method described in Ref. 7. We consider a jellium slab, and we assume that the electron density $n(z)$ vanishes at a distance $z_{0}=2 \lambda_{F}\left(\lambda_{F}=2 \pi / k_{F}\right.$ is the bulk Fermi wavelength $)$ from either jellium edge $\stackrel{32}{ }^{32}$ We expand the single-particle wave functions entering the evaluation of $\chi^{0}\left(q_{\|}, z, z^{\prime}, \omega\right)$ in a sine Fourier representation, and the density-response functions $\chi^{0}\left(q_{\|}, z, z^{\prime}, \omega\right)$ and $\chi_{\lambda}\left(z, z^{\prime} ; q_{\|}, \omega\right)$ in a doublecosine Fourier representation. We also expand the Dirac 
delta function entering Eq. (10) in a double-cosine representation (see Eq. (A2) of Ref. 7). We take all the occupied and unoccupied single-particle orbitals and energies to be the LSDA eigenfunctions and eigenvalues of a KS Hamiltonian, as obtained by using the Perdew-Wang parametrization ${ }^{33}$ of the Ceperley-Alder xc energy of the uniform electron gas 34

In the calculations presented below, we have considered jellium slabs with several bulk parameters $r_{s}$ and a thickness $a=2.23 \lambda_{F}$ for the positive background. For $r_{s}=2.07$, such slab corresponds to about four atomic layers of $\mathrm{Al}(100)$ and it was used in the wavevector analysis of the RPA ${ }^{35}$ and beyond-RPA ${ }^{20,36}$ xc surface energy.

In Figs. 1 and 2, we show contour plots for the exactexchange hole density and the self-consistent RPA xc hole density, respectively. In the bulk, both the exchangeonly hole and the xc hole are spherical and the xc hole is more localized, as in the case of a uniform electron gas. Near the surface, both the exchange-only hole and the xc hole happen to be distorted, the center of gravity being closer to the surface when correlation is included. For an electron that is localized far outside the surface, the corresponding exchange-only hole and xc hole remain localized near the surface; Figs. 1 and 2 show that the introduction of correlation results in a flatter hole, which in the case of an electron that is infinitely far from the surface becomes completely localized at a plane parallel to the surface. This is the image plane. We recall that the RPA xc hole density is exact in the limit of large separations (where $u=\left|\mathbf{r}-\mathbf{r}^{\prime}\right| \rightarrow \infty$ ), and yields therefore the exact location of the image plane.

The integration of the xc hole density over the whole surface plane,

$$
b_{x c}\left([n], z, z^{\prime}\right)=\int_{0}^{\infty} d r \bar{n}_{x c}\left([n] ; r, z, z^{\prime}\right)
$$

represents a quantity of interest for a variety of theoretical and experimental situations (see for example Refs. 37,38). Below we show that $b_{x c}\left([n] ; z, z^{\prime}\right)$ represents a suitable quantity to describe the behavior of the $\mathrm{xc}$ hole corresponding to a given electron located at an arbitrary distance from the surface. In Fig. 3, we plot this quantity, versus $z^{\prime}$, for $r_{s}=2.07$ and a given electron located at $z=0.5 \lambda_{F}, z=0, z=-0.5 \lambda_{F}$, and $z=-1.5 \lambda_{F}$. We see from this figure that (i) correlation damps out the oscillations that the exchange hole exhibits in the bulk part of the surface, and (ii) in the case of a given electron located far from the surface into the vacuum the main part of the exchange-only and the $\mathrm{xc}$ hole is found to be near the surface (see also Figs. 1 and 2), although the exchange-only hole appears to be much more delocalized with a considerable weight within the bulk.

Let us now focus on the on-top xc hole. The LSDA accurately accounts for short wavelength contributions to the xc energy $\stackrel{30}{3}$ thus, all the nonempirical approximations of the xc hole have been constructed to recover the LSDA on-top xc hole $\bar{n}_{x c}^{L S D A}(\mathbf{r}, \mathbf{r})$. The slowly-varying
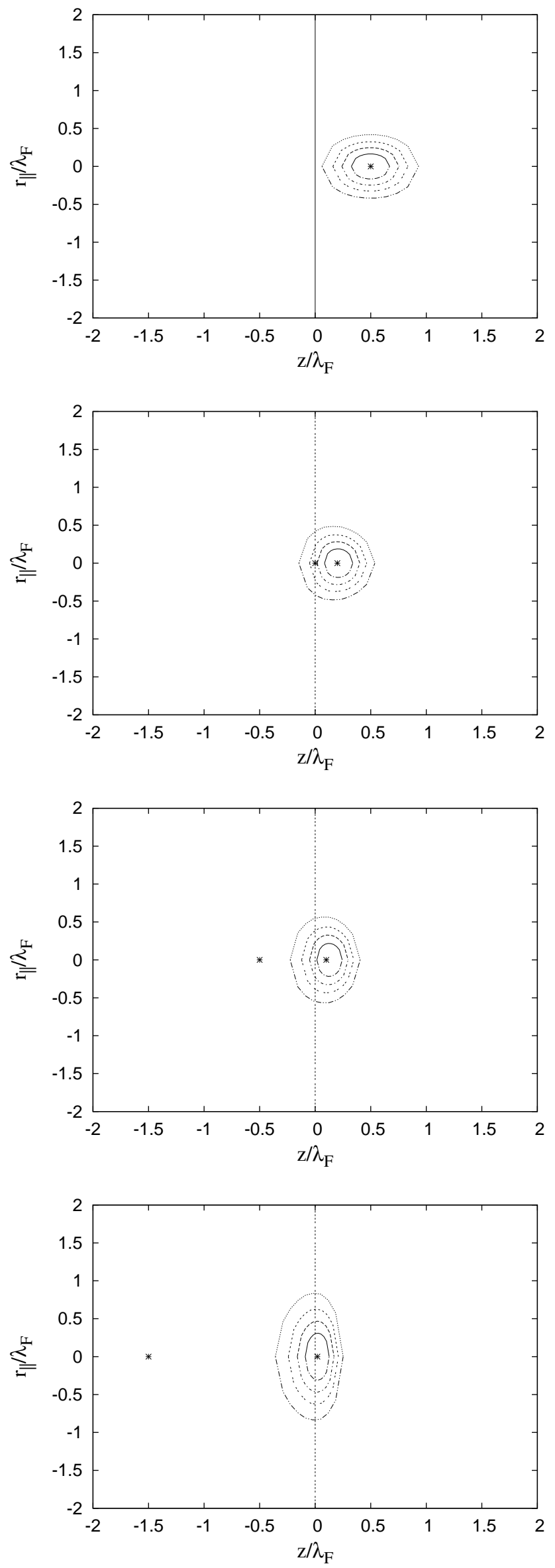

FIG. 1: Contour plots of the exchange hole density $\bar{n}_{x}\left(r_{\|}, z, z^{\prime}\right)$ for several fixed values of the electron position: $z=0.5 \lambda_{F}$ (inside the bulk), $z=0$ (on the surface), 

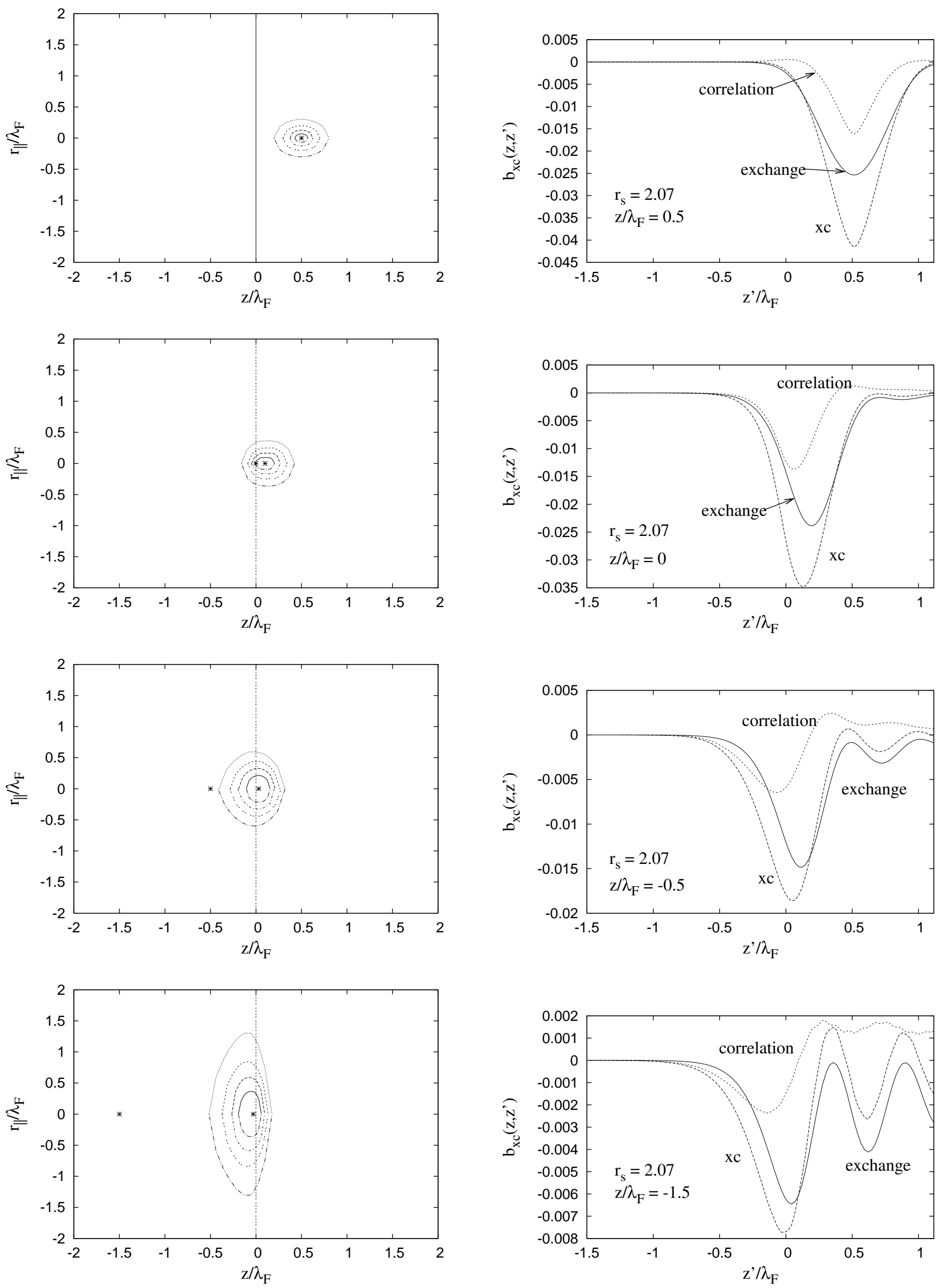

FIG. 2: Contour plots of the RPA coupling-constantaveraged xc hole density $\bar{n}_{x}\left(r_{\|}, z, z^{\prime}\right)$ for several fixed values of the electron position: $z=0.5 \lambda_{F}$ (inside the bulk), $z=0$ (on

FIG. 3: $b_{x c}\left(z, z^{\prime}\right)$ of Eq. (11) versus $z^{\prime} / \lambda_{F}$ for the same positions of the electron as in Figs. 1 and 2, The bulk parameter is $r_{s}=2.07$ and the jellium surface is at $z=0$. 


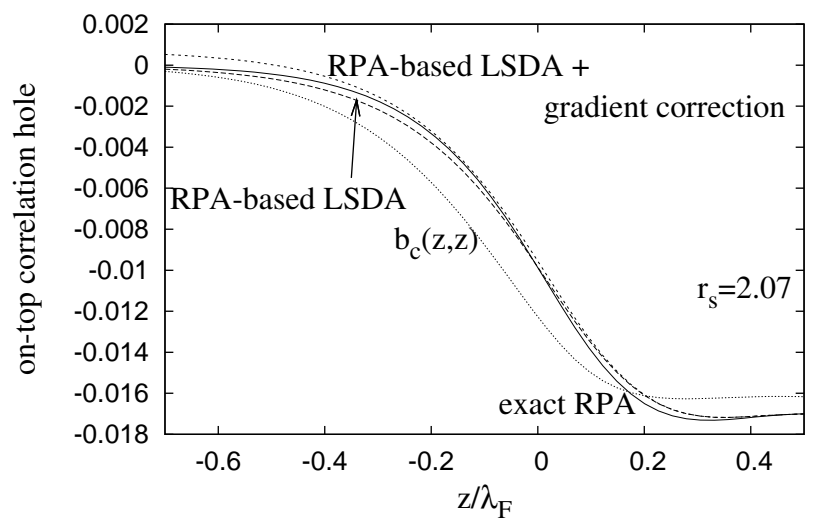

FIG. 4: On-top coupling-constant-averaged correlation hole $\bar{n}_{c}(\mathbf{r}, \mathbf{r})$ at a jellium surface. Also shown is $b_{c}(z, z)$ of Eq. (11). The bulk parameter is $r_{s}=2.07$ and the jellium surface is at $z=0$.

electron gas was treated within RPA by Langreth and Perdew ${ }^{5}$. For a spin-unpolarized system, the gradient correction to the LSDA on-top correlaton hole density is 31

$$
\bar{n}_{c}^{G E A}(\mathbf{r}, \mathbf{r})=\bar{n}_{c}^{L S D A}(\mathbf{r}, \mathbf{r})+\frac{|\nabla n|^{2}}{72 \pi^{3} n^{2}} .
$$

In Fig. 4] we show the on-top correlation hole for the exact RPA, the RPA-based LSDA (see Ref. 15) and the RPA-based GEA of Eq. (12). We see that for a jellium surface the RPA-based LSDA on-top correlation hole nearly coincides with the corresponding exact RPA on-top correlation hole; this is in contrast with the case of strong inhomogeneous systems (e.g., Hooke's atom) $\underline{\underline{30}}$ The gradient correction of Eq. (12) improves the already accurate RPA-based LSDA on-top correlation hole in the slowly-varying density region, but is inacurate in the tail of the density. Fig. 4 also shows that the integrated $b_{c}(z, z)$ of Eq. (11) is more (less) negative in the vacuum (bulk) than the actual on-top correlation hole.

At this point, we would like to emphasize that while the RPA on-top correlation hole in the bulk is too negative but finite, the on-top correlation hole diverges in the bulk within a TDDFT scheme that uses a wavevector and frequency independet xc kernel like in the adiabatic local-density approximation (ALDA)

$$
f_{x c, \lambda}^{A L D A}[n]\left(\mathbf{r}, \mathbf{r}^{\prime}, \omega\right)=\frac{d v_{x c}^{\lambda, \text { unif }}[n(\mathbf{r})]}{d n(\mathbf{r})} \delta\left(\mathbf{r}-\mathbf{r}^{\prime}\right),
$$

or the energy-optimized local-density approximation of Ref. 39. (See the discussion after Eq. (3.9) of Ref. 39). Here, $v_{x c}^{\lambda, \text { unif }}[n(\mathbf{r})]$ is the xc potential of a uniform electron gas of density $n(\mathbf{r})$. An xc kernel borrowed from a uniform-gas xc kernel that has the correct largewavevector behavior (see, e.g., the xc kernels of Refs. 40, 41 42) would yield a finite on-top correlation hole. Fig.

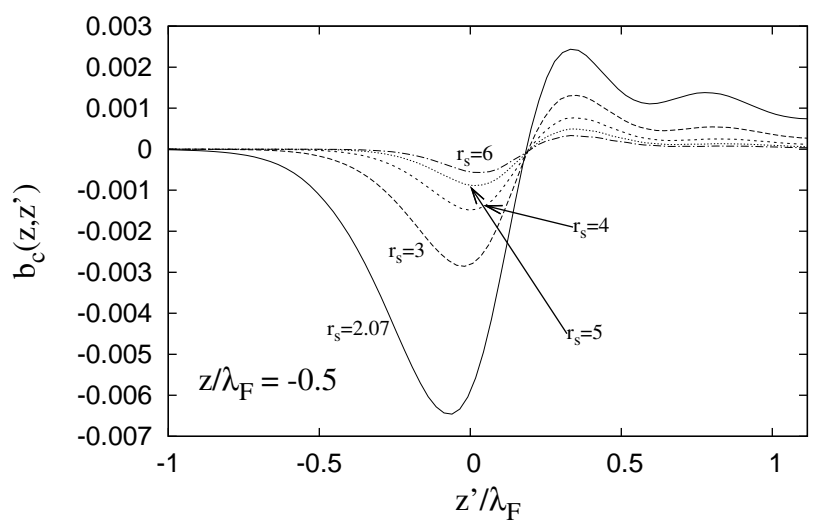

FIG. 5: The correlation hole $b_{c}\left(z, z^{\prime}\right)$ of an electron at position $z=-0.5 \lambda_{F}$ for several values of the bulk parameter $r_{s}=2.07,3,4,5$, and 6 . The jellium surface is at $z=0$.

5) shows the integrated correlation hole of Eq. (11) for an electron at the vacuum side of the surface, at the position $z=-0.5 \lambda_{F}$ and for several values of the electrondensity parameter $r_{s}: 1.5,2.07,3,4,5$, and 6 . In the bulk, the correlation hole exhibits damped oscillations with $r_{s}$-dependent amplitude and a period that does not depend on the electron density and is close to the period $\left(\sim 0.56 \lambda_{F}\right)$ of the corresponding oscillations exhibited by the exchange-only hole.

Finally, we look at the xc energy density $e_{x c}$ defined in Eq. (3). We note that adding to the actual $e_{x c}$ of Eq. (3) an arbitrary function of the position $\mathbf{r}$ that integrates to zero yields the same total $\mathrm{xc}$ energy ${ }^{43}$ The Laplacian of the density $\nabla^{2} n$ integrates to zero for finite systems, it plays an important role in the gradient expansion of the kinetic-energy density, $\stackrel{44,45,46}{\longleftarrow}$ and it is an important ingredient in the construction of density-functional approximations for the kinetic energy density $\stackrel{44,45}{\underline{4}}$ and the xc energy $\stackrel{45}{\underline{4}}$

We define the simplest possible Laplacian-level RPAbased LSDA (the RPA-based L-LSDA) xc energy density:

$$
e_{x c}^{L-L S D A-R P A}(\mathbf{r})=e_{x c}^{L S D A-R P A}(\mathbf{r})-C \nabla^{2} n(\mathbf{r}),
$$

where $C$ is a constant parameter which we find by minimizing the difference between $e_{x c}^{R P A-L-L S D A}$ and $e_{x c}^{R P A}$. We find $C=0.3$ for a jellium slab with $r_{s}=2.07$, and its value gets larger as $r_{s}$ increases.

In Fig. [6, we show $\Delta e_{x c}(z)=e_{x c}^{R P A}(z)-e_{x c}^{a p p r o x}(z)$ versus $z / \lambda_{F}$ for a jellium slab with $r_{s}=2.07$ and several RPA-based approximations for $e_{x c}^{a p p r o x}(z)$. The RPAbased PBE 15 improves considerably the behavior of the RPA-based LDA. The ARPA-GGA ${ }^{47}$ is a GGA functional that fits the RPA xc energy density of the Airy gas and is remarkably accurate for jellium surfaces. The RPA-based GGA++ is the RPA version of the GGA++ of Ref. 38. $\quad\left(e_{x c}^{R P A-G G A++}=e_{x c}^{R P A-L S D A} F_{x c}(l)\right.$, where $l=r_{s}^{2} \nabla^{2} n / n$ is a reduced Laplacian and $F_{x c}(l)$ is de- 


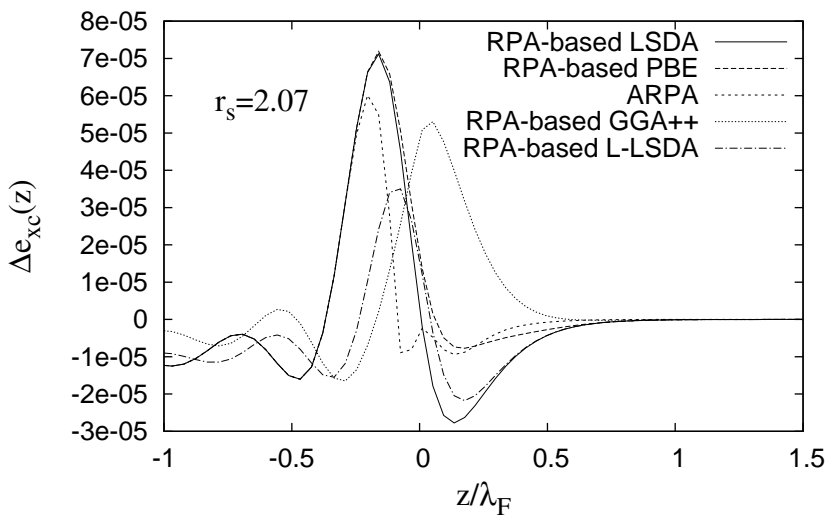

FIG. 6: $\Delta e_{x c}(z)=e_{x c}^{R P A}(z)-e_{x c}^{a p p r o x}(z)$ versus $z / \lambda_{F}$ at a surface of a jellium slab, for several xc approximations: RPAbased LSDA ${ }^{15}$, RPA-based PBE ${ }^{15}$, ARPA GGA ${ }^{47}$, RPAbased GGA $++\frac{38}{2}$, and RPA-based L-LSDA (Eq. (14) with $C=0.3)$. The bulk parameter is $r_{s}=2.07$, and the edge of the positive background is at $z=0$.

fined in Eq. (3) of Ref. 38.) Although the GGA++ functional was constructed for the Si crystal, we observe that the RPA-based GGA++ improves over the RPA-based LSDA in the bulk near the jellium surface showing that it can be a good approximation for systems with small oscillations. (In the bulk, close to the jellium surface, there are Friedel oscillations as well as quantum oscillations due to the finite thickness of the jellium slab). We note finally that $e_{x c}^{R P A-L-L S D A}$ significantly reduces the local error of the RPA-based LSDA near the jellium surface, although by construction $E_{x c}^{R P A-L-L S D A}=E_{x c}^{R P A-L S D A}$.

\section{CONCLUSIONS}

We have presented extensive self-consistent calculations of the exact-exchange hole and the RPA xc hole at a jellium surface.

We have presented a detailed study of the RPA xc hole density at a metal surface. When the electron is in the vacuum, its hole remains localized near the surface (its minimum is on the image plane) and has damped oscillations in the bulk. We find that the on-top correlation hole is accurately described by local and semilocal densityfunctional approximations, as expected from Ref. 5 . We also find that for an electron that is localized far outside the surface the main part of the corresponding xc hole is completely localized at a plane parallel to the surface, which is the image plane.

Because of an integration by parts that occurs in the underlying gradient expansion, a GGA (or meta-GGA) hole is meaningful only after averaging over the electron density $n(\mathbf{r}) \stackrel{18,20}{=}$ This average smooths the sharp cutoffs used in the construction of the angle-averaged GGA xc hole density. The wavevector analysis of the jelium xc surface energy is an important and hard test for the LSDA, GGA, and meta-GGA angle-averaged xc hole densities, showing not only the accuracy of the xc hole but also the error cancellation between their exchange and correlation contributions. Thus, Refs. 35 and 20,21 have shown that the TPSS meta-GGA 21 and the PBEsol $\mathrm{GGA}^{20} \mathrm{xc}$ hole densities improve considerably the accuracy of their LSDA and PBE counterparts at jellium surfaces, both within RPA and beyond RPA. $\underline{48}$

The exchange energy density does not have a gradient expansion $\stackrel{49}{ }$, as does the kinetic energy density. However the existence of gradient expansion of the xc energy density is still an open problem. We use our RPA xc hole density to compare the xc energy densities of several approximations. The most accurate ones are ARPA GGA of Ref. 47 and RPA-based L-LSDA of Eq. (14).

\section{Acknowledgments}

We thank J. P. Perdew and J. F. Dobson for many valuable discussions and suggestions. J.M.P. acknowledges partial support by the Spanish MEC (grant No. FIS2006-01343 and CSD2006-53) and the EC 6th framework Network of Excellence NANOQUANTA. L.A.C. acknowledges NSF support (Grant No. DMR05-01588).
1 Kohn, W.; Sham, L.J. Phys. Rev. 1965, 140, A1133.

2 Perdew, J.P.; Kurth, S. in "A primer in density functional theory", edited by C. Fiolhais, F. Nogueira and M. Marques, p.1 (2003).

3 Callem, H.B.; Welton, T.R. Phys. Rev. 1951, 83, 34 .

4 Harris, J.; Griffin, A. Phys. Rev. B 1975, 11, 3669 .

5 Langreth, D.C.; Perdew, J.P. Phys. Rev. B 1977, 15, 2884 ; 1980, 21, 5469; 1982, 26, 2810.

6 Gunnarsson, O.; Lundqvist, B.I. Phys. Rev. B 1976, 13, 4274 .

7 Pitarke, J.M.; Eguiluz, A.G. Phys. Rev. B 1998, 57, 6329; 2001, 63, 045116.

8 Gross, E.K.U.; Dobson, J.F.; Petersilka, M., in Density
Functional Theory II, Vol.181 of Topics in Current Chemistry, edited by Nalewajski R.F., Springer, Berlin, 1996, p.81.

9 Gross, E.K.U.; Kohn, W. Phys. Rev. Lett. 1985, 55, 2850.

10 Levy, M. Phys. Rev. A 1991, 43, 4637.

11 Levy, M. Bull. Am. Phys. Soc. 1990, 35, 822.

12 Levy, M.; Perdew, J.P. Int. J. of Quantum Chem. 1994, 49, 539.

13 Perdew, J.P.; Schmidt. K. in Density Functional Theory and Its Application to Materials, V. Van Doren et al., American Institute of Physics, Melville, NY, 2001.

14 Ernzerhof, M.; Perdew, J.P. J. Chem. Phys. 1998, 109, 3313. 
15 Yan, Z.; Perdew, J.P.; Kurth, S. Phys. Rev. B 2000, 61, 16430.

16 Perdew, J.P.; Wang, Y. Phys. Rev. B 1992, 46, 12947.

17 Gori-Giorgi, P.; Perdew, J.P. Phys. Rev. B 2002, 66, 165118.

18 Perdew, J.P.; Burke, K.; Wang, Y. Phys. Rev. B 1996, 54, 16533.

19 Perdew, J.P.; Burke, K.; Ernzerhof, M. Phys. Rev. Lett. 1996, 77, 3865 .

20 Constantin, L.A.; Perdew, J.P.; Pitarke, J.M. (unpublished).

21 Constantin, L.A.; Perdew, J.P.; Tao, J. Phys. Rev. B 2006, 73, 205104.

22 Sahni, V.; Bohnen, K.-P. Phys. Rev. B 1984, 29, 1045 ; 1985, 31, 7651.

23 Sahni, V.; Ma, C.Q.; Flamholz, J.S. Phys. Rev. B 1978, $18,3931$.

24 Juretschke, H.J. Phys. Rev. 1953, 92, 1140.

25 Moore, I.D.; March, N.H. Ann. Phys. (N.Y.) 1976, 97, 136.

26 Newns, D.M. Phys. Rev. B 1970, 1, 3304.

27 Inglesfield, J.E.; Moore, I.D. Solid State Comm. 1978, 26, 867.

28 Pitarke, J.M.; Eguiluz, A.G. Bull. Am. Phys. Soc. 1994, 39,$515 ; \mathbf{1 9 9 5}, 40,33$.

29 Nekovee, M.; Pitarke, J.M. Comp. Phys. Commun. 2001, $137,123$.

30 Burke, K.; Perdew, J.P.; Langreth, D.C. Phys. Rev. Lett. 1994, 73, 1283.

31 Burke, K.; Perdew, J.P.; Ernzerhof, M. J. Chem. Phys. 1998, 109, 3760 .

$32 z_{0}=2 \lambda_{F}$ is sufficiently large for the physical results to be accurate.

33 Perdew, J.P.; Wang, Y. Phys. Rev. B 1992, 45, 13244.
34 Ceperley, D.M.; Alder, B.J. Phys. Rev. Lett. 1980, 45, 566.

35 Pitarke, J.M.; Constantin, L.A.; Perdew, J.P. Phys. Rev. $B$ 2006, 74, 045121.

36 Constantin, L.A.; Pitarke, J.M.; Dobson, J.F.; GarciaLekue, A.; Perdew, J.P. Phys. Rev. Lett. 2008, 100, 036401.

37 Nekovee, M.; Foulkes, W.M.; Needs, R.J. Phys. Rev. B 2003, 68, 235108.

38 Cancio, A.C.; Chou, M.Y. Phys. Rev. B 2006, 74, $081202(\mathrm{R})$.

39 Dobson, J.F.; Wang, J. Phys. Rev. B 2000, 62, 10038.

40 Corradini, M.; Del Sole, R,; Onida, G.; Palummo, M. Phys. Rev. B 1998, 57, 14569.

41 Constantin, L.A,; Pitarke, J.M. Phys. Rev. B 2007, 75, 245127.

42 Pitarke, J.M.; Perdew, J.P. Phys. Rev. B 2003, 67, 045101.

43 Tao, J.; Staroverov, V.N.; Scuseria, G.E.; Perdew, J.P. Phys. Rev. A 2008, 77, 012509.

44 Constantin, L.A.; Ruzsinszky, A. submitted to Phys. Rev. $B$

45 Perdew, J.P.; Constantin, L.A. Phys. Rev. B 2007, 75, 155109.

46 Kirzhnitz, D.A. Sov. Phys. JETP 1957, 5, 64; Kirzhnitz, D.A. in Field Theoretical Methods in Many-Body Systems, Pergamon, Oxford, 1967.

47 Constantin, L.A,; Ruzsinszky, A.; Perdew, J.P. to be submitted at Phys. Rev. B.

48 In Ref. ${ }^{20}$, the PBEsol wavevector analysis of a jellium surface had been compared to an accurate TDDFT calculation that used the xc kernel of Ref. ${ }^{42}$.

49 Perdew, J.P.; Wang, Y. in Mathematics Applied to Science, edited by Goldstein, J.A.; Rosencrans, S.; Sod G., Academic, 1988. 